\title{
Effectiveness of Wharton's jelly stem cells in gastroschisis repair using the inner surface of the umbilical cord as a patch: long-term results
}

\author{
Stojan M. Zivkovic ${ }^{a}$, Miroslav Djordjevic ${ }^{a}$, Natasa Milic ${ }^{c}$, Zeljko Smoljanic ${ }^{b}$, \\ Goran Djuricic $^{b}$, Polina Pavicevic ${ }^{b}$, Branislava Keserovic ${ }^{d}$ and \\ Borko Stojanovic $^{a}$
}

\begin{abstract}
Objective This retrospective study was designed to assess and compare the anatomical, functional, and esthetic appearance of the umbilical area in patients after repair of gastroschisis using the inner surface of the umbilical cord (UC) and in patients with omphalocele conservatively treated.
\end{abstract}

Background Our procedure transformed gastroschisis into an 'artificial-surgical omphalocele' in which the prolapsed intestine was covered with an umbilical patch, the inner surface of which contained Wharton's jelly (WJ).

Methods We have summarized an experience of 17 years in repairing gastroschisis using the inner surface of the UC. From 1986 to 2003, 21 infants with gastroschisis and one with a ruptured omphalocele were treated with this technique. We used Eagle's medium to prove the validity of the umbilical stump and the duration of its viability. The inner surface of the umbilical patch is a 'live' structure with WJ, which contains mucoid connective tissue and fibroblast-like cells - that is, stem cells producing cutis, adipose, and connective tissue.

Results Using our method, early control assessment of 18 of 21 patients with gastroschisis, at intervals of 1-3 months, showed good functional and esthetic results.

\section{Introduction}

Many procedures and materials used for gastroschisis repair have been described in the literature. However, most of them failed to meet expectations and were abandoned. An up-to-date standard approach to the reconstruction of this defect was essentially based on using the full thickness of an umbilical cord (UC) [1]. Unfortunately, minimal intervention management (MIM), as well as some other methods and techniques, may successfully solve only simple cases [2]. In complex cases results were mostly negative. Recently, three new procedures and devices for complex cases of gastroschisis reduction have been reported, such as 'spring-loaded silo', 'applied Alexis', and 'negative pressure wound therapy', with a pump for negative pressure and interface kits $[3,4]$. Although all of these techniques have a broad indication field, they can hardly be applied in most underdeveloped countries because of their high price, and sometimes they are unavailable even in developed countries [5]. These devices and materials as foreign bodies and 'dead' materials, cannot benefit from the normal healing process, which yields a scar between the artificial material and surrounding tissue. Some of these procedures are
Clinical long-term results in terms of anatomical, clinical, and functional findings were excellent. Besides clinical testimony, we used high-frequency ultrasonography to make an appraisal of the effectiveness of WJ stem cells in the repair of gastroschisis, and compared our results with healthy volunteers and patients with omphalocele conservatively treated.

Conclusion This paper describes the effect of the local application of WJ - that is, mesenchymal stromal cells derived from the inner surface of the umbilical stump - and its influence on the healing process of the birth defect and wound. Ann Pediatr Surg 11:173-180 (c) 2015 Annals of Pediatric Surgery.

Annals of Pediatric Surgery 2015, 11:173-180

Keywords: gastroschisis, high frequency ultrasound imaging, inner surface of umbilical patch, ruptured omphalocele, Wharton's jelly

aDepartment of Pediatric Surgery, 'bepartment of Pediatric Radiology, University Children's Hospital, 'Institute for Statistics and Informatics, School of Medicine, University of Belgrade and ${ }^{\mathrm{d} T o r l a k}$ Institute of Immunology and Virology, Belgrade, Serbia

Correspondence to Stojan M. Zivkovic, MD, PhD, Department of Pediatric Surgery, University Children's Hospital, Tirsova 10, Belgrade 11000, Serbia Tel/fax: + 38111227 5532; e-mail: stojanmzivkovic@gmail.com

Received 21 June 2013 accepted 22 June 2015

relatively minimally invasive because of the short operating time, but do not always offer benefit [6].

Because of these reasons, the best solution for gastroschisis reduction is the application of an umbilical patch to cover the abdominal defect and wound. The autogenous umbilical stump (US) is always at hand; the patch is nontoxic, nonantigenic and, above all, safe and gratis. In simple and in some complicated cases the umbilical patch with a sterile inner surface that contains Wharton's jelly (WJ) covering the defect and lying on the intestine gives satisfactory and in some cases even excellent results [7]. Enlargement of the defect and augmentation of the abdominal cavity may be very effective in managing some of the difficult gastroschisis cases. At the same time, WJ facilitates maximal regeneration of skin, adipose, connective, and contractile cells.

In this paper we describe our long-term results with the inner surface of the umbilical cord (ISUC), and the effect of $\mathrm{WJ}$ on the functional and esthetic appearance of the umbilical area after the reconstruction of gastroschisis. In comparison, conservative treatment of omphalocele or other techniques for gastroschisis reduction using full 
thickness - that is, the external amnion layer of the UC or a patch of lyophilized dura mater - do not have the same degree of benefit.

\section{Patients and methods}

Our single-stage procedure of covering the prolapsed intestine and defect with the sterile ISUG was aimed to transform gastroschisis into a 'surgical-artificial' (S-A) omphalocele. Treatment was continued in a conservative manner in true omphalocele using povidone. Twenty-one patients with gastroschisis were treated with our technique from 1986 to 2003. A small abdominal defect, for safe reduction, required an enlargement in 13 patients. Only in five patients was the widening of the abdominal wall defect not performed. In three patients, two with gastroschisis and one with a ruptured omphalocele, the reduction was impracticable. In these patients treatment was converted to a new method in which a part of the intestine and US were placed into the abdomen and the remaining intestine into a sterile plastic bag.

At the same time, 15 of 26 patients with omphalocele were treated conservatively. All of them had a temporary ventral hernia, which was closed in nine cases. Data on four out of six patients were available for statistical analysis.

\section{Operative technique}

Our method and operative technique were previously described in detail [7], although some elements were not mentioned. Reduction was performed without anesthesia and analgesia but with a nonpharmacological sedation [8,9]. Manual stretching of the abdominal wall and defect allowed the reduction of the majority of eviscerated intestines. If the defect was too small for the reduction of the intestine, it could be enlarged laterally by incision of the rim of the fascial defect on the right side. Reduction of most of the eviscerated intestine into the abdomen was feasible after a mild stretching of the abdominal defect and wall (Fig. 1).

Before opening the UC and formatting a patch an appraisal of the feasibility of reduction should be carried out. The mild manual stretching of the abdominal defect and wall will allow the reduction of most of the eviscerated intestine into the abdomen. If reduction is impossible, the next step should be conversion to a new method: namely, one part of the intestine and US is placed into the abdominal cavity for preservation of the US, whereas the rest of the intestine is placed into a plastic bag.

\section{Preservation of the umbilical cord 'in vivo' for complex gastroschisis}

Our simple technique cannot be performed in complex cases of gastroschisis with huge viscera-abdominal disproportion or intestinal atresia and huge peel surface. However, management of such cases can be converted to placing a part of the intestine and the US into the abdomen and the remaining intestine into a plastic bag for 7-10 days.

Conversion was performed in three cases: in two cases of gastroschisis, one with intestinal atresia and another with a significant visceral disproportion and peel; and in a patient with a ruptured omphalocele with the intestine and the spleen protruding out of the abdominal cavity. Conversion was successful in all of them. The stump was still viable and both the defect and the intestine were covered with the inner surface of the umbilical patch. Therefore, delayed application and creation of the umbilical patch were feasible. Thereafter, the US was opened longitudinally, and the defect and the intestine were covered by formatting an S-A omphalocele, as described earlier. Further treatment continued conservatively as in the case of the true omphalocele (Fig. 2).

\section{Culture of the umbilical sample}

There are several articles in the literature describing the significance of WJ that reveal that these cells possess ultrastructural characteristics of both fibroblasts and smooth muscle cells, indicating that they are modified, rather than being typical fibroblasts [10]. Gastroschisis patients with varying stump lengths were received by our department $2-10 \mathrm{~h}$ after delivery. Similarly to the studies conducted by Takechi and Kuwabara [10], our objective was to prove the vitality and durability of the US after delivery using Eagle's medium for tissue culture. To this end, six full-term UC samples measuring $2-3 \mathrm{~cm}$ were collected and processed within $1-10 \mathrm{~h}$ after normal delivery. Eagle's medium was poured into the UC segments using tissue culture flasks (Table 1).

Samples were incubated at $37^{\circ} \mathrm{C}$ for $3-5$ days and cells from WJ showed rapid proliferation. Five of six stumps in the tissue culture flasks showed proliferation and adhesion to the plastic surface. The oldest one showed infection. Therefore, our aim to prove the viability of WJ cells was successfully achieved. This experiment showed clearly that $10 \mathrm{~h}$ after delivery the UC tissue was alive and capable of growing and developing. The umbilical patch produces 'in vivo' strong 'symphysis' with the defect and the wound [7,9], and restores the skin, subcutaneous adipose and connective tissue, which is visible later on high-frequency (HF) ultrasound.

\section{High-frequency ultrasound image}

We used HF ultrasound as a noninvasive tool to evaluate some parameters among healthy volunteers, in patients whose gastroschisis defect was engrafted with ISUC and WJ and in patients with omphaloceles conservatively treated (Fig. 3). Ultrasound scanning provides a reliable morphological representation of the skin in horizontal echogenic lines (Fig. 3b). Below the skin there was a layer of hypoechoic subcutaneous fat, which was followed by deep fascia, bilateral rectus abdominis muscle, and finally the abdominal cavity [11]. Echograms of the skin, subcutaneous tissue, and the deeper structures were detected using 10,12 , and $15 \mathrm{MHz}$ transducers. Measurements (DICOM) of the intramuscular distance (IMD) between two inner ends of the rectus abdominis muscles were taken for all patients. Thicknesses of the skin and subcutaneous adipose tissue were measured from the inner rim of the right rectus muscle in healthy volunteers and in patients with right-sided gastroschisis. Skin measurements for the true omphalocele were 
Fig. 1
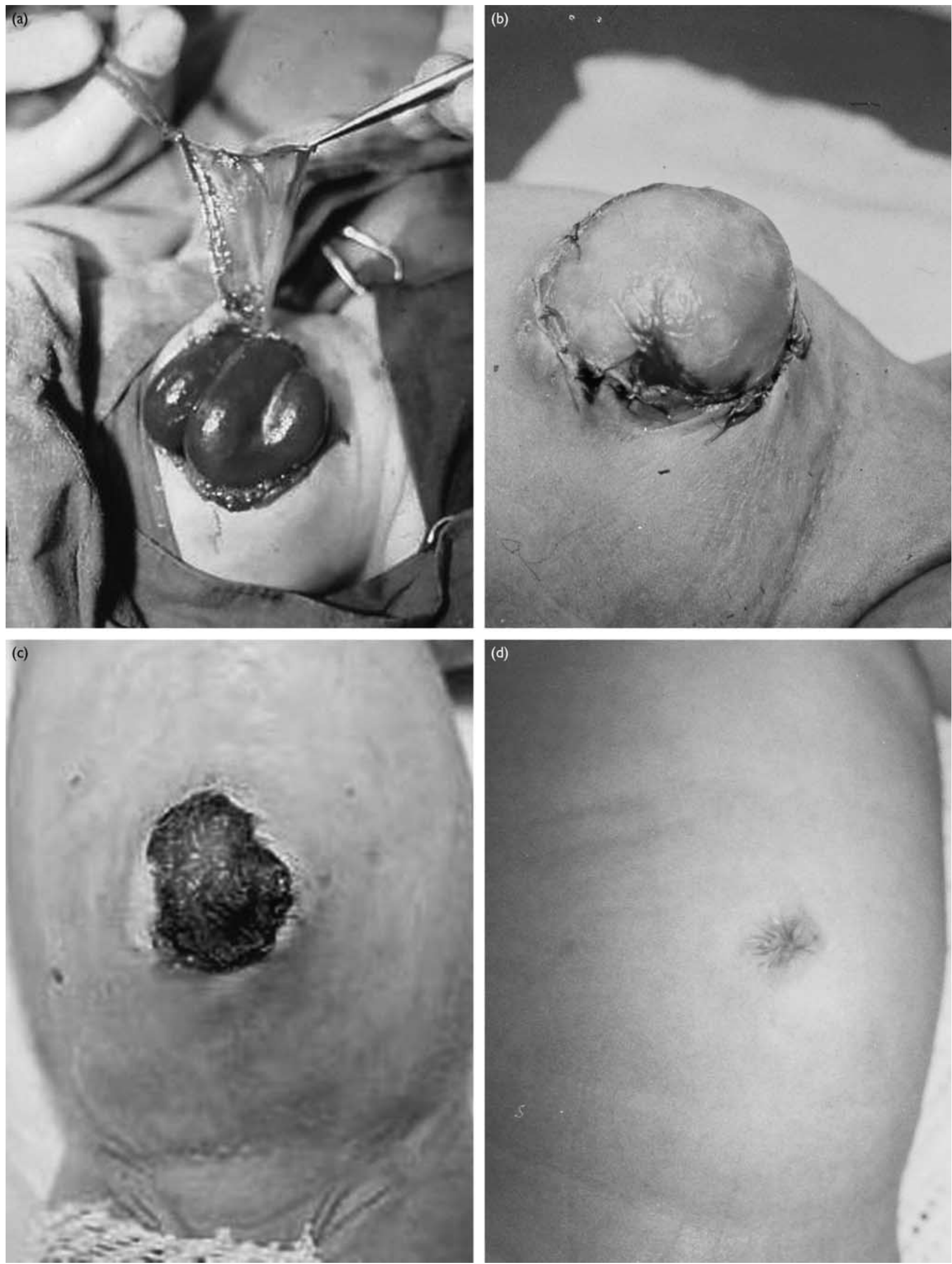

Our operative method and early result. (a) After stretching the abdominal wall and enlargement of the gastroschisis defect, reduction of most of the eviscerated intestine into the abdomen is feasible. The inner surface of the umbilical patch, which contains Wharton's jelly, is grafted over the intestine and defect. (b) The umbilical patch may cover a defect $\sim 4 \times 5 \mathrm{~cm}$,depending on the length of the umbilical stump. This surgical-artificial omphalocele showed symphysis between the patch, skin, and wound of the gastroschisis defect. (c) Necrosis of the external surface of the umbilical patch - amnion - was treated with povidone. (d) Early result of engrafting Wharton's jelly with the inner surface of the umbilical patch.

Copyright (C) 2015 Annals of Pediatric Surgery. Unauthorized reproduction of this article is prohibited. 
Fig. 2
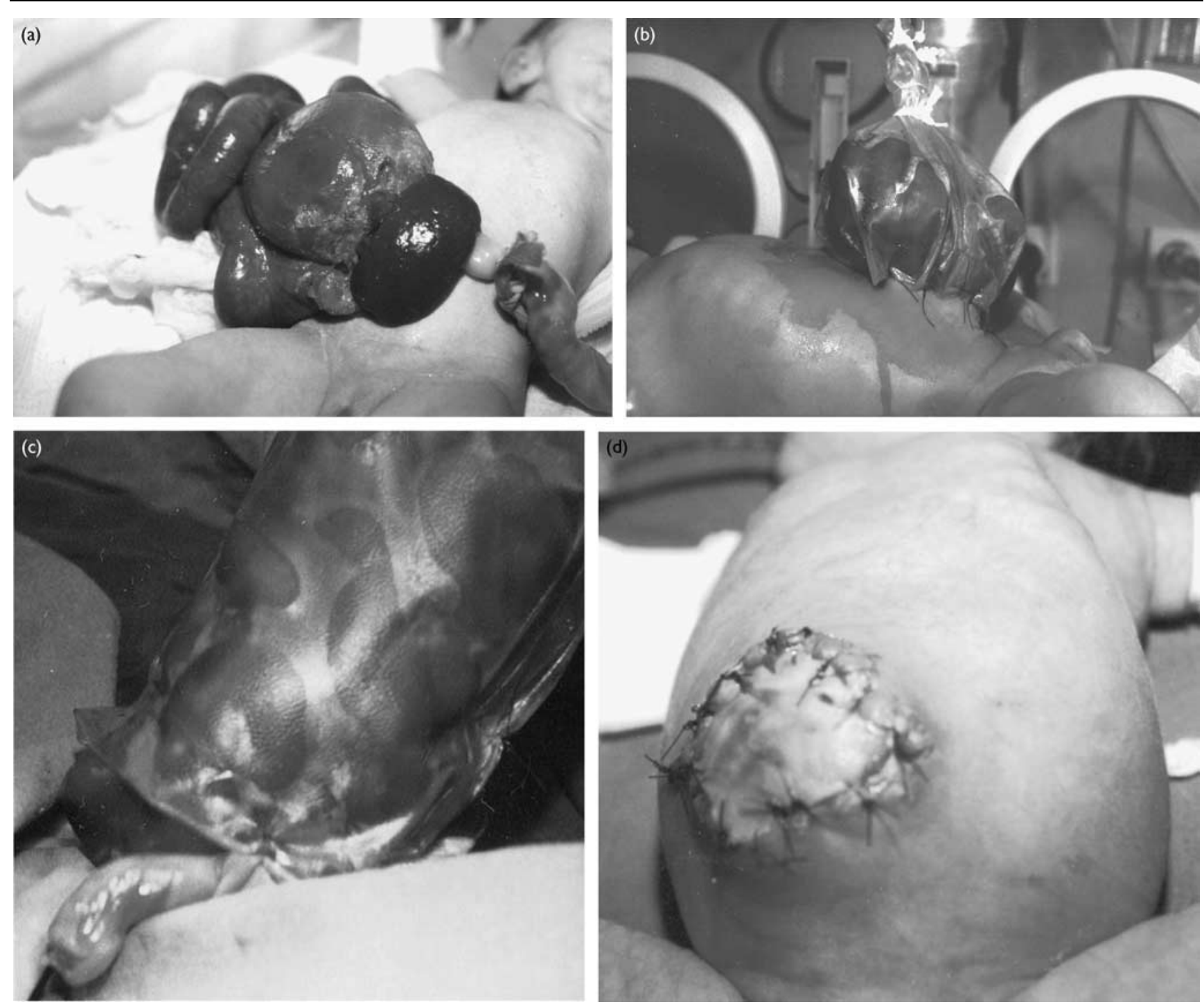

Conversion of a ruptured omphalocele into a 'surgical-artificial' omphalocele. (a) Ruptured omphalocele with stomach, small and large intestine, ovary, and spleen outside the abdomen. (b) The spleen, umbilical stump, and a part of the intestine returned partially to the abdominal cavity. (c) After ten days in the abdominal cavity and plastic bag, the umbilical stump looks clinically normal and fresh. (d) From umbilical stump created patch and its inner portion-surface is engrafted to close the defect.

Table 1 Umbilical stumps in Eagle's medium

\begin{tabular}{|c|c|c|c|c|c|}
\hline Mothers' initials & Patient sex & Delivery date, time & Time of sampling & Age of sample & Proliferation in Eagle's medium \\
\hline J.D. & Male & 13 June $1997,12: 25 \mathrm{~h}$ & $20: 30 \mathrm{~h}$ & $7: 30 \mathrm{~h}$ & + \\
\hline M.S. & Female & 13 June 1997, 10:50 h & $20: 40 \mathrm{~h}$ & $9: 50 \mathrm{~h}$ & + \\
\hline P.M. & Male & 13 June $1997,11: 20 \mathrm{~h}$ & $21: 00 \mathrm{~h}$ & $9: 49 \mathrm{~h}$ & Infection \\
\hline J.P. & Female & 14 June $1997,13: 30 \mathrm{~h}$ & $20: 20 \mathrm{~h}$ & $7: 50 \mathrm{~h}$ & + \\
\hline P.S. & Female & 14 June $1997,19: 40 \mathrm{~h}$ & $20: 30 \mathrm{~h}$ & $50 \mathrm{~min}$ & + \\
\hline D.A. & Female & 14 June $1997,19: 55 \mathrm{~h}$ & $21: 00 \mathrm{~h}$ & $1: 5 \mathrm{~h}$ & + \\
\hline
\end{tabular}

performed at the very center of the anomaly - that is at the site of the hernia.

\section{Statistical analysis}

Statistical analysis was performed using the statistical package for the social sciences (SPSS, release 17.0 for Windows; SPSS Inc., Chicago, Illinois, USA). Descriptive statistics have been presented as mean values with SD; 95\% confidence interval for the mean values has been presented in parentheses. Follow-up data are presented as median with range. Differences between groups were compared with the parametric Student's test for unpaired data. In all tests, $P$ values less than 0.05 were considered to be statistically significant. 
Fig. 3
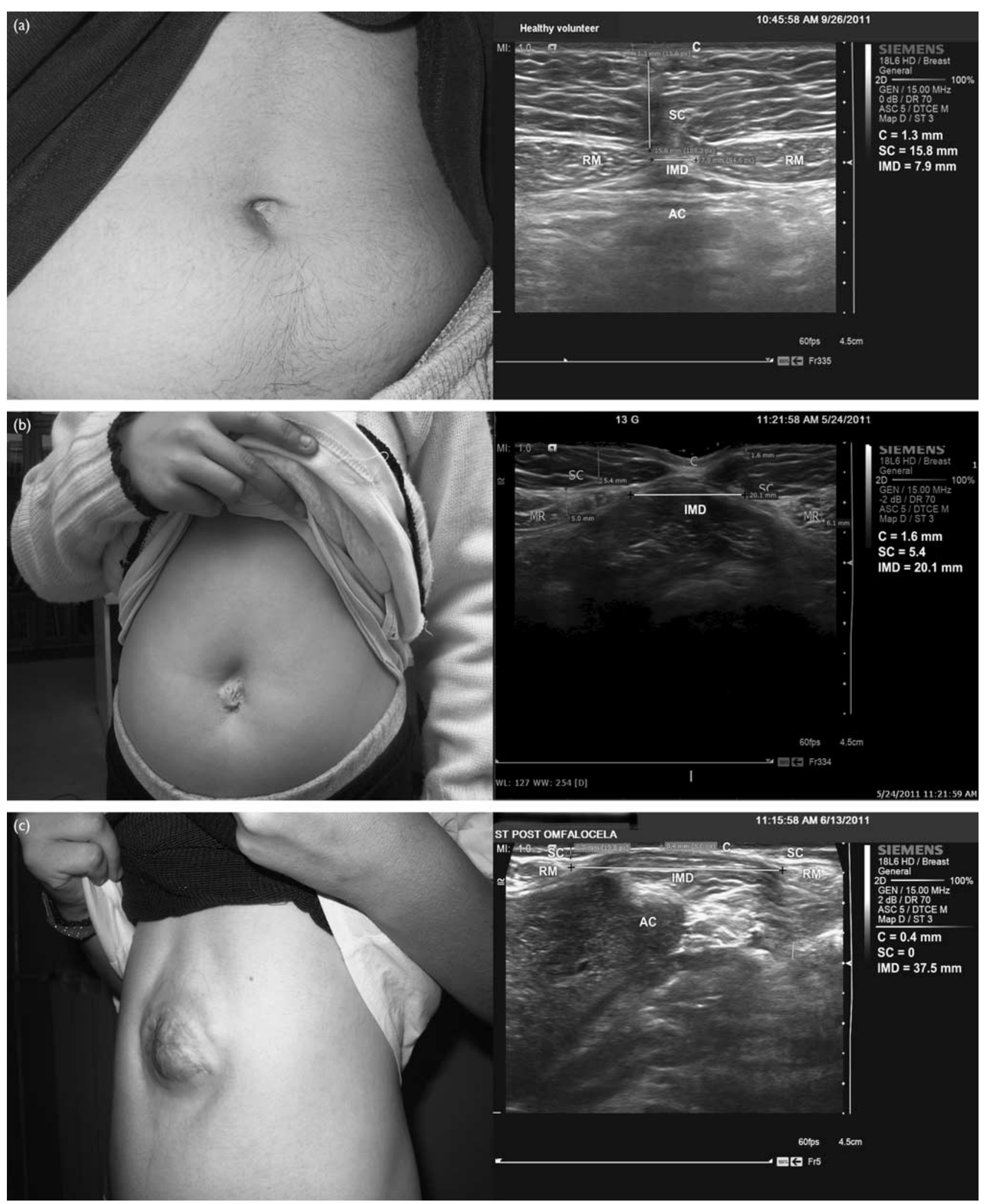

Clinical appearance and high-frequency (HF) ultrasound. (a) Late clinical appearance and HF ultrasound of the umbilical area in a healthy volunteer. Left: a healthy 14-year-old boy with normal appearance of the umbilical area. Right: transverse $15 \mathrm{MHz}$ ultrasound image - cutis skin $1.3 \mathrm{~mm}$, subcutis adipose tissue $15.8 \mathrm{~mm}$, and intramuscular distance (IMD) $7.9 \mathrm{~mm}$. (b) Late clinical appearance and HF ultrasound of the umbilical area in a patient with gastroschisis. Left: clinical aspect of the umbilical area in a 13-year-old girl without enlargement of the umbilical defect. No hernia or small umbilical defect was noted. Right: transverse $15 \mathrm{MHz}$ HF ultrasound - cutis $1.6 \mathrm{~mm}$, Subcutis (SC) $5.6 \mathrm{~mm}$, IMD $26.1 \mathrm{~mm}$. (c) Late clinical appearance and HF ultrasound of the umbilical area in a patient with omphalocele. Left: conservatively treated omphalocele; note, umbilical hernia and diastasis separation of rectus abdominis muscles. Right: transverse $15 \mathrm{MHz}$ image - cutis $0.8-1.8 \mathrm{~mm}$, adipose tissue missing, IMD between both muscles at the umbilical area $37.5 \mathrm{~mm}$. 


\section{Results}

From 1986 to 2003, 21 infants with gastroschisis and one with a ruptured omphalocele were treated with our technique. Small abdominal wall defects required enlargement in $13(61.9 \%)$ patients, whereas in five $(23.8 \%)$ patients the abdominal defect was suitable for intestinal reduction, and in three $(14.28 \%)$ patients a conversion was required. Mortality occurred in three (14.28\%) patients: two with a simple gastroschisis and one with a complex converted gastroschisis. Complications during the first 6 months of this procedure were detected in three patients: one suffered from necrotizing enterocolitis; another had intestinal obstruction caused by a mesenteric window due to trauma during vaginal delivery or reduction; and the third had an edema of the abdominal wall after vigorous stretching, but without any other consequences. In all of our patients, with or without enlargement, umbilical hernia had not been clinically detected during early control assessments. At long-term clinical control assessments, HF ultrasonography, and imaging, six patients showed an absence of even a small fascial defect or hernia. Clinical control assessments and measurements of the umbilical area among gastroschisis patients treated with WJ and among healthy volunteers showed no difference in cutis (skin appendages, hairs) and subcutaneous tissue (Figs $3 a$ and 4b).

Six gastroschisis patients, with a median follow-up time of 15.5 years (range 13-25 years), compared with seven healthy volunteers, with a median follow-up time of 17 years (range 13-24 years), showed normal clinical anatomy of the umbilical area during control evaluations. Results of the HF ultrasonography are presented in Table 2.

There was no statistically significant difference in cutis and subcutis measurements between gastroschisis patients and healthy volunteers $(P>0.05)$. Statistically significant difference was found in IMD measurements between gastroschisis patients and healthy volunteers $(P=0.003)$. Comparison of gastroschisis $+\mathrm{WJ}-$ that is, S-A omphalocele - and true omphalocele with median follow-up time of 14.5 years (range 13-24 years) is presented in Table 3. Note that in omphalocele, adipose tissue is missing in the subcutis. Statistically significant difference in cutis and IMD measurements was found between the gastroschisis and omphalocele group $(P=0.016$ and 0.002 , respectively) (Fig. $3 \mathrm{~b}$ and $\mathrm{c})$.

\section{Discussion}

Our method in two-thirds of our patients required augmentation of the abdominal cavity by mild stretching of the abdominal defect and the anterior wall. In longitudinal opening the US, we created a patch that could be engrafted onto defects sized $4 \times 5 \mathrm{~cm}$ or even larger, depending on the length of the stump. Closure with this sterile patch of ISUC, which contains the WJ, cannot be compared with closure with MIM with full thickness of the umbilicus - that is, umbilical amnion or with closure using a patch of the lyophilized dura mater, as in both the cases the WJ is missing, and the full thickness of the umbilicus covers the intestine with the outer layer - that is, umbilical amnion of the US [12].
This technique (MIM) was accepted later and followed sporadically in our hospital. External layer of UC is not sterile and passing through birth canal can result in infection. Explanation for two patients with high temperature of an unknown cause postoperatively, and also umbilical and inguinal hernia. This MIM with the full thickness of the umbilicus covers a considerably smaller area. Comparative effectiveness of the inner surface of the US with Wharton's jelly and external layer of US - amnion, is clearly visible in Fig. 4.

In our practice, we performed transformation of gastroschisis into an S-A omphalocele, where the inner surface of the US was engrafted onto the intestine. This primary closure does not require a complete reduction of the intestine. The rest of the intestine is covered with the viable umbilical patch. This patch is a 'safety valve', and in addition to the augmentation of the abdominal cavity it may prevent many well-known complications. Our procedure is suitable for patients with not so large visceroabdominal disproportion, peel, atresia, and a narrow gastroschisis defect. This patch prevents adhesion with the underlying bowel, and separation and tearing of the patch have never been recorded. The umbilical patch proved to be solid, and any kind of strengthening during the procedure was not needed. Reinforcement of the defect with man-made material results in ventral hernias in $60-62 \%$ of cases after closure, and a hernia repair is required in $21 \%$ [2,13,14].

A comparison of clinical and $\mathrm{HF}$ ultrasound imaging findings between treatment with gastroschisis $+\mathrm{WJ}$ and omphalocele (omphalocele conservatively treated) + amnion clearly showed the effectiveness of WJ stem cells (Fig. 3b and c). In gastroschisis $+\mathrm{WJ}$, the full thickness of the skin and subcutaneous tissue reaches the navel, that is umbilical shadow on HF ultrasound. Contrary to omphalocele - amniocele conservatively treated have thin, skin scar without dermis, skin appendages and sucutaneous adipose tissue on the whole surface of affected area, and not to the navel.

\section{About Wharton's jelly in the light of our technique}

In a study by Takechi and Kuwabara [10] to determine the significance of $\mathrm{WJ}$, the characteristics of these cells were examined by means of electron microscopy and immunohistochemistry. These cells possessed ultrastructural characteristics of both fibroblasts and smooth muscle cells, indicating that they are modified, rather than being typical fibroblasts. As the results of this study showed, these cells can function in both fibrogenesis and cell contraction [10]. Patients coming to our hospital from secondary institutions needed transportation, stabilization, and intestinal reduction $\sim 3-7 \mathrm{~h}$ after birth. Our experiment clearly showed that $10 \mathrm{~h}$ after delivery the UC tissue was alive and capable of growing in Eagle's medium. WJ of the UC contains mucoid connective tissue and fibroblast-like cells. This gelatinous connective tissue gives the cord resiliency and pliability, and protects the blood vessels in the UC from compression. The UC matrix, called WJ, was previously shown to be a valuable source of mesenchymal stromal cells (MSCs). 

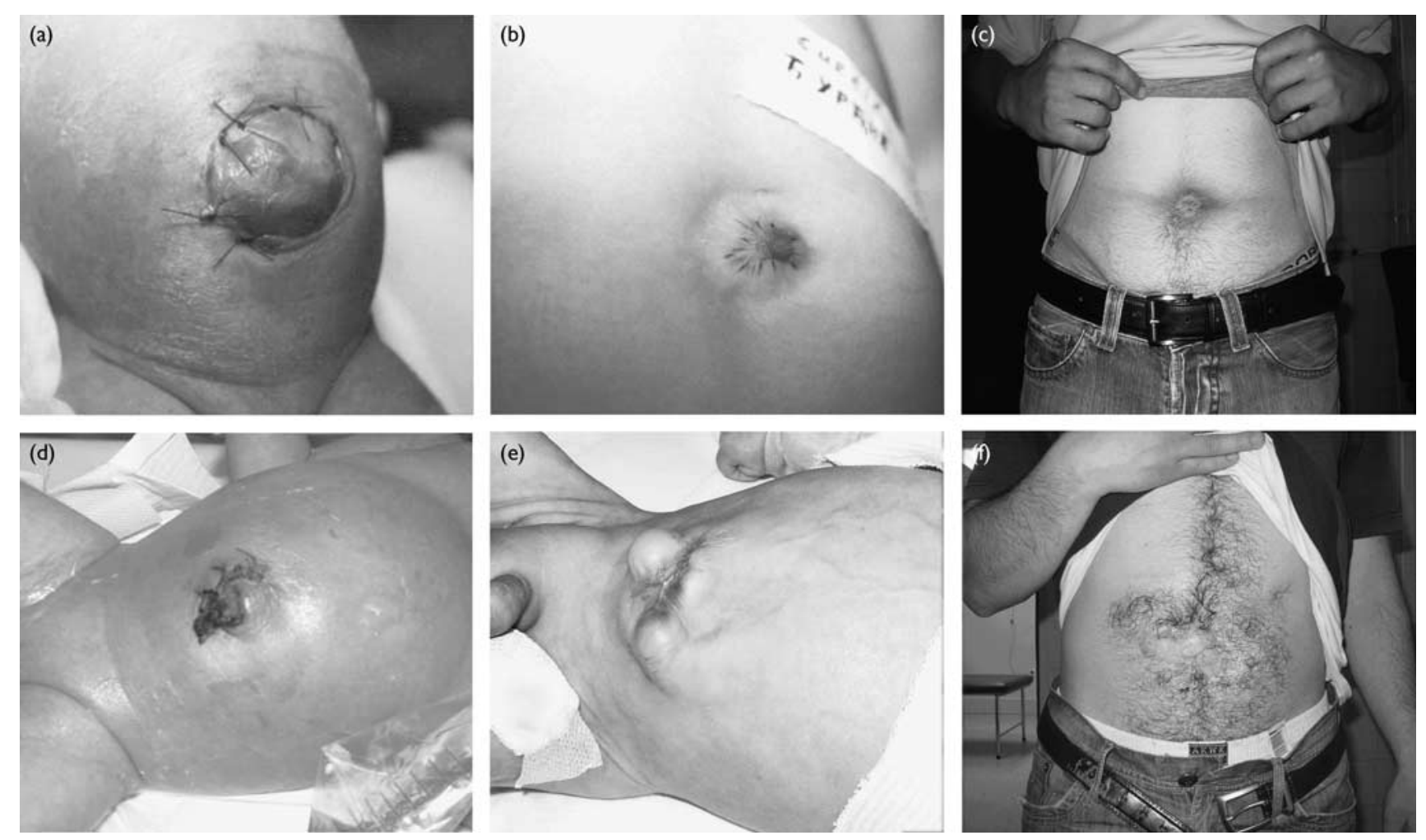

Effectiveness of treatment with the inner surface of the umbilical stump with Wharton's jelly versus external surface of the umbilicus, that is umbilical amnion. Covering with Wharton's jelly: (a) intestine covered with internal portion of the umbilical stump and Wharton's jelly; (b) appearance 3 months after the procedure; (c) umbilical area with hair as a result of stem cells responsible for hair production. Covering with umbilical amnion: (d) intestine covered with external layer of umbilicus (umbilical amnion) in minimal intervention management (MIM) procedure; (e) unusual umbilical finding after 'MIM', bulging area represents hernias; (f) umbilical area covered with thin skin without hair.

Table 2 Cutis, subcutis, and intramuscular distance measurements for gastroschisis patients and healthy volunteers

\begin{tabular}{lllc}
\hline Variables & Gastroschisis + WJ $(n=6)$ & Healthy volunteers $(n=7)$ & $P$ \\
\hline Cutis & $1.48 \pm 0.377(1.06-1.89)$ & $1.68 \pm 0.343(1.35-2.02)$ & $>0.05$ \\
Subcutis & $11.1 \pm 1.74(7.36-14.94)$ & $9.18 \pm 3.94(6.09-12.28)$ & $>0.05$ \\
IMD & $16.8 \pm 5.29(12.77-20.73)$ & $7.87 \pm 2.15(4.86-10.88)$ & 0.003
\end{tabular}

Data are presented as mean \pm SD; $95 \%$ confidence interval for mean is given in parentheses.

IMD, intramuscular distance; WJ, Wharton's jelly.

Table 3 Cutis and intramuscular distance measurements for gastroschisis and omphalocele patients

\begin{tabular}{llcc}
\hline Variables & Gastroschisis + WJ $(n=6)$ & Omphalocele + amnion $(n=4)$ & $P$ \\
\hline Cutis & $1.48 \pm 0.377(1.06-1.89)$ & $0.633 \pm 0.153(0.12-1.14)$ & 0.016 \\
IMD & $16.8 \pm 5.29(12.77-20.73)$ & $39.5 \pm 7.00(32.20-46.75)$ & 0.002
\end{tabular}

Data are presented as mean \pm SD; $95 \%$ confidence interval for mean is given in parentheses.

IMD, intramuscular distance; WJ, Wharton's jelly.

Using flow cytometric analysis some authors have isolated mesenchymal cells from the UC that express matrix receptors and integrin markers.

These cells have multilineage potential and, under suitable culture conditions, are able to differentiate into cells of the adipogenic and osteogenic lineages $[15,16]$.

However, isolation and expansion of cells from human full-term UCs may be performed using the WJ without enzyme digestion or dissection. The procedure was based on the plastic adhesion capacity of MSCs. The expanded cells expressed characteristic markers and presented typical functional properties of MSCs, such as differentiation, immunomodulation, and hematopoietic supportive functions [17].

Our experiment to prove the viability of cells from the US was successful and we noted adhesions to tissue culture flasks as the first of three criteria to define MSCs. In 1997, we were not aware of the criteria for defining the viability of MSCs: namely, adhesion to plastic, specific surface antigen expression, and multipotent differentiation potential [18]. In the contemporary literature, expansion of cells from the UG in Eagle's medium and plastic adherence are a well-described and well-established property of MSCs. The human UC is an excellent source of stem cells, which may be therapeutically used in autologous transplantations [19]. In vivo, the basic principle of stem cell therapy is that undifferentiated cells, following delivery to the injured host and migration to the site of injury, will, under the influence of local signals, differentiate to cells of the appropriate phenotype. These differentiated cells then contribute to the repair of the defective tissue. There is evidence to indicate that this is the case, but there are few or no data on the specific signals that give rise to differentiation in situ [20]. Therefore, the sterile ISUC, which contains WJ cells, implanted in a gastroschisis defect stimulates 
the formation of new skin, adipose tissue, and deep fascia that can be assessed both clinically and ultrasonographically (Fig. 4c). At present, it is known that stem cells are responsible for hair production, and hair follicles in different areas of the body produce hairs of different lengths [21]. In contrast, the amniotic epithelial cells from umbilical amnion do not possess such characteristics [12,22] (Fig. 4f).

Mesenchymal stem cells possess immunomodulatory properties and are known to be incapable of tumorigenesis. There are many other important characteristics of mesenchymal stem cells in adults and newborns. For example, these cells do not require feeders; they possess plasticity and multipotency; their lifespan in vitro is short; ethical implications are less significant; and clinical applications are possible [23]. In addition, these cells can inhibit the growth of tumor cells [24].

We also evaluated the possibility of preserving the US 'in vivo' in the abdominal cavity - that is, 'in situ'. The abdominal cavity may be the natural habitat for the UC, where it may remain under the influence of hormones and growth factors, which usually occur 'in vivo'. These features create the possibility for a delayed application of the US after 7-10 days.

\section{Conclusion}

In the absence of available material for gastroschisis closure or due to the discrepancy between the size of the defect and the length of the intestine, we used the US as the patch for engraftment of the abdominal defect. Our study represents the first clinical unintentional autologous transplant of MSCs from WJ in gastroschisis patients, which locally proliferates and differentiates into skin, adipose, and connective tissues. This biological method being free of cost and easily accessible may be a substitute for other very expensive procedures.

\section{Acknowledgements}

The authors thank Dr Jay Prosser, University of Leeds, for English grammar correction, and Milan T. Jovanovic for technical support.

\section{Conflicts of interest}

There are no conflicts of interest.

\section{References}

1 Bianchi A, Dickson AP. Elective delayed reduction and no anesthesia: 'minimal intervention management' for gastroschisis. J Pediatr Surg 1998; 33:1338-1340.
2 Orion KC, Krein M, Liao J, Shaaban AF, Pitcher GJ, Shilyansky J. Outcomes of plastic closure in gastroschisis. Surgery $2011 ; 150: 177-185$.

3 Jensen RA, Waldhausen JHT, Kim SS. The use of a spring-loaded silo for gastroschisis. Arch Surg 2009; 144:516-519.

4 Gabriel A, Gollin G. Management of complicated gastroschisis with porcine small intestinal submucosa and negative pressure wound therapy. J Pediatr Surg 2006; 41:1836-1840.

5 Kusafuka J, Yamataka A, Okazaki T, Okawada M, Urao M, Lane GJ, Miyano T. Gastroschisis reduction using 'Applied Alexis', a wound protector and retractor. Pediatr Surg Int 2005; 21:925-927.

6 Lobo JD, Kim AC, Davis RP, Segura BJ, Alpert H, Teitelbaum DH, et al. No free ride? The hidden costs of delayed operative management using a spring-loaded silo for gastroschisis. J Pediatr Surg 2010; 45:1426-1432.

7 Zivkovic SM. Repair of gastroschisis using umbilical cord as a patch. J Pediatr Surg 1991; 26:1179-1180.

8 Zivkovic SM. Original reconstructive technique for gastroschisis repair using umbilical cord as a patch. Health and Science. Medical Informations. (in Spanish). Abrilde 1992; ano II (No 2):1;18-19.

9 Zivkovic SM. Repair of gastroschisis using umbilical cord as a patch: further experience. Rass Ital Chir Pediatr 1995; 38:187-190.

10 Takechi K, Kuwabara Y. Ultrastructural and immunohistochemical studies of Wharton's jelly umbilical cord cells. Placenta 1993; 14:235-245.

11 Valle M, Zamorani P. Skin and subcutaneous tissue. In: Bianchi S, Martinoli C, editors. Ultrasound of the musculoskeletal system. Berlin, Heidelberg, New York: Springer; 2007. p. 25.

12 Han YM, Romero R, Kim JS, Tarca AL, Kim SK, Draghici S, et al. Region-specific gene expression profiling: novel evidence for biological heterogeneity of the human amnion. Biol Reprod 2008; 79:954-961.

13 Hernández Siverio N, López-Tomassetti Fernández E, Mario Troyano Luque J. Gastroschisis: primary closure using umbilical cord strengthened by polypropylen mesh. J Perinat Med 2007; 35:249-251.

14 Sandler A, Lawrence J, Meehan J, Phearman L, Soper R. A 'Plastic' sutureless abdominal wall closure in gastroschisis. J Pediatr Surg 2004; 39:738-741.

15 Wang HS, Hung SC, Peng ST, Huang CC, Wei HM, Guo YJ, et al. Mesenchymal stem cells in the Wharton's jelly of the human umbilical cord. Stem Cells 2004; 22:1330-1337.

16 Romanov YA, Svintsitskaya VA, Smirnov VN. Searching for alternative sources of postnatal human mesenchymal stem cells: candidate MSC-like cells from umbilical cord. Stem Cells 2003; 21:105-110.

17 De Bruyn C, Najar M, Raicevic G, Meuleman N, Pieters K, Stamatopoulos B, et al. A rapid, simple, and reproducible method for isolation of mesenchymal stromal cells from Wharton's jelly without enzymatic treatment. Stem Cells Dev 2001; 20:547-557.

18 Dominici M, Le Blanc K, Mueller I, Slaper-Cortenbach I, Marini F, Krause D, et al. Minimal criteria for defining multipotent mesenchymal stromal cells. The International Society for Cellular Therapy position statement. Cytotherapy 2006; 8:315-317.

19 Petsa A, Gargani S, Felesakis A, Grigoriadis N, Grigoriadis A. Effectiveness of protocol for the isolation of Wharton's Jelly stem cells in large-scale applications. In Vitro Cell Dev Biol Anim 2009; 45:573-576.

20 Barry FP, Murphy JM. Mesenchymal stem cells: clinical applications and biological characterization. Int J Biochem Cell Biol 2004; 36:568-584.

21 Schneider RK, Püllen A, Kramann R, Bornemann J, Knüchel R, Neuss S, Perez-Bouza A. Long-term survival and characterisation of human umbilical cord-derived mesenchymal stem cells on dermal equivalents. Differentiation 2010; 79:182-193.

22 Rennekampff HO, Dohrmann P, Föry R, Fändrich F. Evaluation of amniotic membrane as adhesion prophylaxis in a novel surgical gastroschisis model. J Invest Surg 1994; 7:187-193.

23 Pozzobon M, Ghionzoli M, De Coppi P. ES, iPS, MSC, and AFS cells. Stem cells exploitation for pediatric surgery: current research and perspective. Pediatr Surg Int 2010; 26:3-10.

24 Qiao L, Xu Z, Zhao T, Zhao Z, Shi M, Zhao RC, et al. Suppression of tumorigenesis by human mesenchymal stem cells in a hepatoma model. Cell Res 2008; 18:500-507. 